\title{
有机反应机制的准经典分子动态学研究进展
}

\author{
张凯瑞王亚亚朱宏丹彭 谦* \\ (南开大学化学学院 元素有机化学国家重点实验室 天津 300071)
}

\begin{abstract}
摘要 准经典分子动态学模拟方法结合了经典分子动力学和电子结构理论, 从原子/分子层面研究化学反应的动态机 制, 不但能模拟反应体系中相应产物或中间体的统计学分布，还可提供时间尺度下化学键生成/断裂的信息. 密度泛函 理论(DFT)计算在反应机制研究中已被广泛应用, 但从准经典分子动态学角度的研究还相对较少, 比如分叉过渡态的 现象及其选择性、环加成协同机制中出现的分步过程以及绕过常见的中间体而直接生成产物等. 这些新颖机制的研究 通常需要分子动态学, 有些甚至打破了传统过渡态理论的认知. 综述了近年来有机化学反应机制的准经典分子动态学 研究进展, 强调机制中的动态学效应, 旨在加深人们对有机反应机制的理解并拓宽有机化学理论.
\end{abstract}

关键词 准经典分子动态学; 分叉过渡态; 协同机制; 分步机制; 动态学效应

\section{Advances on Quasi-classical Molecular Dynamics of Organic Reaction Mechanisms}

\author{
Zhang, Kairui Wang, Yaya Zhu, Hongdan Peng, Qian* \\ (State Key Laboratory of Elemento-Organic Chemistry, College of Chemistry, Nankai University, Tianjin 300071)
}

\begin{abstract}
Quasi-classical molecular dynamics is a computational method that combines classical molecular dynamics and electronic structure theory, which can not only simulate the statistics of the corresponding products or intermediates in the reaction mechanism, but also provide dynamic information of chemical bond formation/cleavage on time scales. Density functional theory (DFT) calculation has been widely used in the research of reaction mechanism, but there are relatively few studies from the perspective of quasi-classical molecular dynamics, such as the phenomenon of bifurcations of transition states and their selectivity, stepwise processes appear in the concerted mechanism of cycloaddition, bypassing common intermediates and directly generating products, etc. These novel mechanism processes often require molecular dynamics, and some even break the cognition of traditional transition state theory. The recent research progress of quasi-classical molecular dynamics of organic chemical reaction mechanisms is reviewed, with emphasis on the dynamic effect in the mechanism, in order to deepening people's understanding of organic reaction mechanisms and broaden the theory of organic chemistry.
\end{abstract}

Keywords quasi-classical molecular dynamic; bifurcation of transition state; concerted mechanism; stepwise mechanism; dynamic effect

在 20 世纪 60 年代, 化学家们已经发展出早期的分 子动力学(Molecular Dynamics) $)^{[1-2]}$, 并将其应用于简单 化学反应, 帮助人们在原子/分子层级理解化学微观状 态. 1963 年, Bunker ${ }^{[3]}$ 报道了用蒙特卡洛方法计算三原 子分子 $\mathrm{N}_{2} \mathrm{O}$ 和 $\mathrm{O}_{3}$ 解离速率. 密度泛函理论(Density Fun-

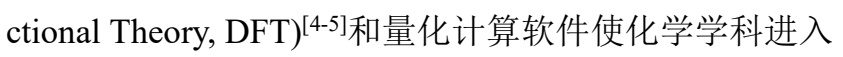

到理论与实验相互配合研究的新阶段, 被广泛应用于物 理、化学以及材料等领域. DFT 方法因其高精度以及适 度的计算成本, 在有机化学反应机制研究中发挥着重要 作用, 可计算出过渡态和中间体等静态点组成的反应势 能面, 但这样的机制描述缺少化学反应中的分子运动轨 迹信息. 张东辉等 ${ }^{[6]}$ 在分子反应动力学领域做出重要贡

\footnotetext{
* Corresponding author. E-mail: qpeng@nankai.edu.cn

Received February 20, 2021; revised March 18, 2021; published online March 25, 2021.

Dedicated to the 100th anniversary of Chemistry at Nankai University.

Project supported by the National Natural Science Foundation of China (Nos. 21890722, 21950410519, 21702109), the Natural Science Foundation of Tianjin Municipality (Nos. 19JCJQJC62300, 18JCYBJC21400), the Tianjin Research Innovation Project for Postgraduate Students (No. 2019YJSB081) and the Fundamental Research Funds for Central Universities (Nankai University).

国家自然科学基金(Nos. 21890722, 21950410519, 21702109)、天津自然科学基金(Nos. 19JCJQJC62300, 18JCYBJC21400)、天津研究生科研创新计划(No. 2019YJSB081)和中央高校基本科研基金(南开大学)资助项目.
} 
献，发展了基于高精度势能面的量子动力学模拟计算方 法, 其计算精度可以和杨学明等 ${ }^{[7]}$ 的仪器观测相互验证, 共同推动研究不断深入. 然而高精度的计算成本昂贵, 现阶段还很难应用于复杂有机体系的反应机制研究.

在物理化学中, 热力学(Thermodynamics)用来判断 化学反应的方向及其可行性, 动力学(Kinetics)则用来研 究反应进行的速率和反应机理, 与活化能直接相关. 而 在本文中, 动态学更强调时间尺度上的分子运动轨迹. 虽然人们也将 dynamics 译为动力学(如经典分子动力 学), 本文在前言中沿用这一用法, 但在正文及后续部 分, 为了避免混淆, 涉及含时分子运动轨迹时称动态学, 而涉及活化能垒时称动力学. 准经典分子动态学 ${ }^{[8-9]}$ 是 在经典分子动力学的基础上结合了电子结构理论, 从该 理论信息中直接读取动态学模拟所需的势能、梯度和 Hessian 矩阵等作为轨迹的初始条件. 准经典分子动态 学中的力源于电子结构理论, 在实际计算中, 对于由 $N$ 个原子组成的孤立体系, 原子的运动由牛顿经典运动方 程决定 ${ }^{[10]}$ :

$$
F\left(r_{1}, r_{2}, \ldots r_{N}\right)=\sum_{i} m_{i} a_{i}=\sum_{i} m_{i} \frac{d^{2} r_{i}}{d t^{2}}
$$

$F$ 是作用于原子的力, $m_{i}$ 是原子的质量, $a_{i}$ 是原子的加速 度, $t$ 为时间. 力也可以表示为负的势能梯度:

$$
F_{i}=m_{i} \frac{d^{2} r_{i}}{d t^{2}}=-\frac{d U\left(r_{i}\right)}{d r_{i}}
$$

$U$ 是体系的势能, 牛顿运动方程可以将势能的导数与位 置随时间的变化联系起来. 一般的有机体系通常包含几 十到几百个原子, 进行动态学模拟时需对所有原子的运 动方程进行积分, 此处介绍的 Verlet 是目前最常用的算 法之一, 它使用时间 $t$ 处的位置 $r$ 和加速度 $a$ 以及时间 $t-\delta t$ 中的位置来计算时间 $t+\delta t$ 处的新位置,

$$
\begin{aligned}
& r(t+\delta t)=r(t)+v(t) \delta t+\frac{1}{2} a(t) \delta t^{2} \\
& r(t+\delta t)=2 r(t)-r(t-\delta t)+a(t) \delta t^{2}
\end{aligned}
$$

这有别于传统的分子动力学 (Molecular Dynamics,

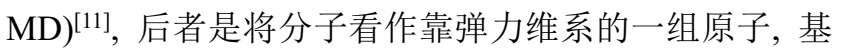
于牛顿力学和经验参数 (如力场参数) 计算分子运动, 故 计算速度快, 已被广泛应用于生物大分子如蛋白体系. 由于不求解电子结构, 分子动力学很难描述具备丰富化 学键形成和断裂等过程的反应机制研究. 而准经典分子 动态学不仅可以研究分子动态行为, 还可获得时间尺度 下化学键变化等结构信息, 因此可用于研究复杂有机体 系的反应机理.

1985 年, Car 和 Parrinello ${ }^{[12]}$ 提出了重要的卡尔-帕
里内洛分子动力学(Car-Parrinello Molecular Dynamics, CPMD). 该方法只优化一步波函数，把波函数看作和原 子核相互作用的具有虚拟质量的电子云，并计算其受 力, 得到下一步的坐标位置. 但是 CPMD 只能用于基态 下的分子运动体系，且需约 $0.1 \mathrm{fs}$ 的计算时间步长，大 大限制了其应用. 尽管如此，由于当时计算机算力低， 较大体系的 DFT 计算非常昂贵, 能实现基于电子结构 的分子动力学模拟是相当重要的突破.

1990 年, Helgaker 和 Uggerud 等 ${ }^{[13]}$ 首次报道了玻恩奥本海默分子动力学(Born-Oppenheimer molecular dynamics, BOMD)模型的应用. 它是基于 Born-Oppenheimer 近似计算指定系统基态能量和结构的分子动力 学模拟手段 ${ }^{[14-15]}$, 可直接通过 Gaussian 接口使用, 无需 另外安装其他程序. BOMD 在每一步动力学计算中都要 计算基态电子能量和原子核的受力, 在求解运动方程时 可以使用时间尺度较大的步长 $(1 \sim 10 \mathrm{fs})$. 但相较于其 他准经典分子动态学组件, BOMD 计算所花费的时间成 本仍然较高.

目前, 发展较为成熟的准经典分子动态学组件有以 下几种. 1996 年 Hase 等[16]发展了 VENUS 组件，可以和 Gaussian $^{[17]}$, NWChem ${ }^{[18]}$ 和 MOPAC ${ }^{[19]}$ 联用, 其课题组也 应用该方法研究了经典反应机理的动态学行为, 如 $\mathrm{S}_{\mathrm{N}} 2$ 反应等. 2003 年, Singleton 等[20]发展的与 Gaussian 联用 的 Progdyn 组件现在已经成为了研究分叉过渡态现象等 领域的行业标准. 尽管如此, Progdyn 缺少运行记录, 初 学者自行排查计算出错原因较为困难. 2018 年, Ess 等 ${ }^{[21]}$ 发展了与 Gaussian 联用的 DynSuite 组件用于准经典分 子动态学研究. DynSuite 以 Progdyn 为蓝本, 添加了日志 系统用于错误检查及信息记录，使用起来更加高效、方 便. 此外, GAMESS ${ }^{[22]}$ 也可以进行准经典分子动态学模 拟.

准经典分子动态学在解释分叉过渡态选择性(包括 环加成反应、 $\sigma$-重排反应和碳正离子重排等)、理解反应 动态协同-分步机制和动态学效应等方面有着重要的应 用, 这些研究均有赖于对化学键振动时间尺度的认识. 例如我们可计算出化学键的振动频率 $v$, 进而计算出振 动周期 $\tau$ :

$$
\tau=\frac{1}{v c}
$$

其中 $c$ 为光速 $\left(3.0 \times 10^{8} \mathrm{~m} / \mathrm{s}\right)$, 由此可得出 $\mathrm{C}-\mathrm{C}$ 键、 $\mathrm{C}-$ $\mathrm{H}$ 键和 $\mathrm{O}-\mathrm{H}$ 键的振动周期大概范围分别为 $30 \sim 60$ 、 $10 \sim 12$ 和 $8 \sim 11 \mathrm{fs}$. 据此我们可以近似区分出化学键振 动与原子运动成键以及相应断键过程. 本文针对有机化 学反应过程, 综述了近年来准经典分子动态学视角的机 理理解. 


\section{1 分叉过渡态选择性的动态学研究}

在分叉过渡态 ${ }^{[23]}$ 的研究中, 准经典分子动态学发 挥着至关重要的作用. 过渡态理论(Transition State Theory, TST)描述了化学反应中反应物和产物之间较高能 量的过渡状态, 这种状态对应着该势能面上的局部能量 极大值和其他方向上极小值(鞍点区域), 传统上, 过渡 态仅能导向一种中间体. 但是, 如果化学反应中存在着 后过渡态分叉 ${ }^{[24]}$ (Post-transition-state Bifurcation, PTSB), 过渡态可能连接着两种或两种以上的中间体(图 1). 分 叉现象往往出现在两个顺序出现的过渡态之间没有局 部能量极小值的状态下, 此时势能面上连接两个过渡态 的反应路径产生分叉, 分叉点被称作谷脊拐点 (Valley-ridge Inflection, VRI), 这与过渡态理论不同, 其 选择性不能通过活化能来判断, 而是受势能面形状和动 态学效应控制. 因此, 需要使用准经典分子动态学来研 究分叉过渡态的选择性问题.

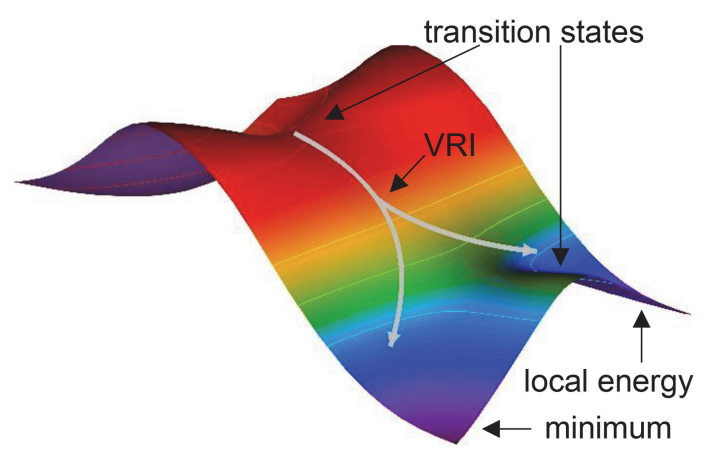

图 1 分叉过渡态势能面

Figure 1 A bifurcating potential energy surface

\section{1 环加成反应}

2002 年, Caramella 等[25]在环戊二烯二聚的环加成 反应中, 首次揭示了周环反应的分叉过渡态现象. 如图 2 所示, 作者在研究发现, 势能面中过渡态 TS-2 是双自 由基形式的一阶鞍点, 通过协同方式生成环加成产物 3 . 尽管 TS-2 保持着 $C 2$ 对称性, 但随着反应坐标向 Cope TS-4 移动, 受 Cope TS-4 虚频对应的反对称 C-C 振动 影响, 过渡态分叉产生了两种反应路径, 导向了两种不 同的 $\mathrm{C}-\mathrm{C}$ 成键方式, 尽管最终生成产物的分子结构是 完全相同的. 作者通过反应坐标扫描首次确定了类似图 2 中的分叉过渡态现象, 为进一步理论和实验研究提供 重要基础.

涉及分叉过渡态的反应，其整体选择性可能会受到 动态学或动力学的控制. 当分子运动轨迹的比例决定产 物分布时, 反应由动态学控制; 而当生成产物的动力学

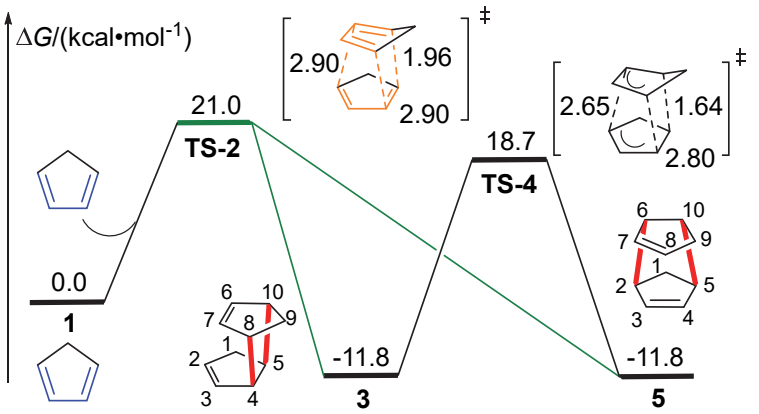

图 2 环戊二烯二聚反应势能面

Figure 2 Free energy profiles for the dimerization of cyclopentadiene

All calculations were performed using B3LYP/6-31G* level of theory. The bond lengths are in angstroms

能垒决定产物分布时, 反应由动力学控制. 2015 年, 余 志祥和王少仲等 ${ }^{[26]}$ 报道了金催化扩环和螺环化反应的 实验和理论研究. 如图 3 所示, $\mathrm{C}(1)$ 位酯基取代的反应 物 6 经分叉过渡态 TS-7, 生成了可由[1,5]-烯基迁移转 化的中间体 9 和 11 , 二者互相转化的能垒高于其分别转 化为产物 10 和 12 的能垒(图中曲线上所标注能量是反 应过程中动力学能垒最高的过渡态的能量). 此时两种 中间体的相互转化比扩环和螺环化慢, 产物分布等同于 二者的动态学分叉比例, 因此作者认为该反应受动态学 控制. 而在 C(1)位甲基取代的反应物 6'对应催化体系 中, 中间体之间的相互转化比扩环和螺环化更快, 此时 反应受动态学的影响大大减弱, 反应选择性将受动力学 控制生成动力学能垒低 $9.4 \mathrm{kcal} / \mathrm{mol}$ 的扩环产物. 该工 作首次发现了同一反应系统中同时存在动态学/动力学 控制分叉势能面选择性的多种情况.

尽管分叉过渡态的发现不需要引入准经典分子动 态学研究, 但动态学在研究过渡态分叉选择性以及取代 基的影响等方面都有着不可替代的作用. 2019 年, Burns 课题组 ${ }^{[27]}$ 报道了 $1,3-$ - 二烯和氧化吡啶鎓叶立德环加成 反应的动态学研究. 如图 4 所示, TS-15 的反应路径 (Intrinsic reaction coordinate, IRC)计算仅导向[4+3]环加 成产物 16 , 而反应的自由能能垒 $(16.1 \mathrm{kcal} / \mathrm{mol})$ 远低于 经过 TS-17 的[3,3]- $\sigma$ 重排反应能垒 $(>30 \mathrm{kcal} / \mathrm{mol})$, 较 高的活化能阻止了两种产物之间的自由转化, 这样的理 论预测无法解释实验中的 $[5+2]$ 环加成产物 18 . 进一步 的准经典分子动态学模拟 $\mathrm{BOMD}$ (步长 $0.25 \mathrm{amu} 1 / 2 \cdot \mathrm{bohr}$, 共 200 步)研究表明, 该反应产物的分布由环加成分叉 步直接确定. 从 TS-15 附近启动的轨迹平均经过 $89 \mathrm{fs}$ 生成[4+3]环加成产物 16 和[5+2]环加成产物 18, 预测 比例为 $1: 0.9$, 与实验观察到的产物比例 $1: 0.88$ 高度 一致. 该反应直接受反应动态学控制而没有经过 $[3,3]-\sigma$ 重排过程. 此外, 作者还讨论了 Krenske 和 Harmata 等 ${ }^{[28]}$ 报道的相关 $[4+3]$ 环加成反应没有出现 $[5+2]$ 环加 


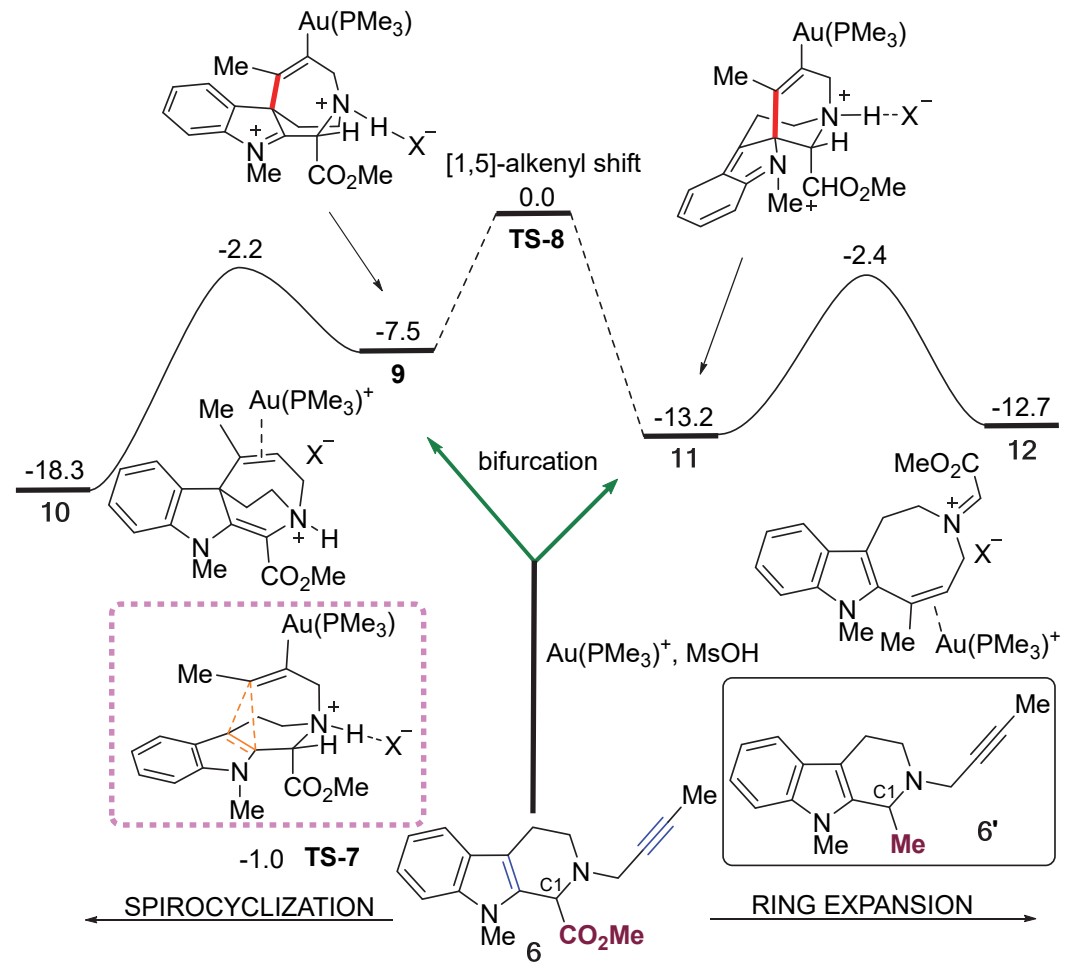

图 3 金催化扩环和螺环化反应的势能面

Figure 3 Free energy profiles for gold-catalyzed ring expansion and spirocyclization reactions QM calculations were performed using B3LYP/6-31G*(SDD for Au)//SMD (toluene) level of theory

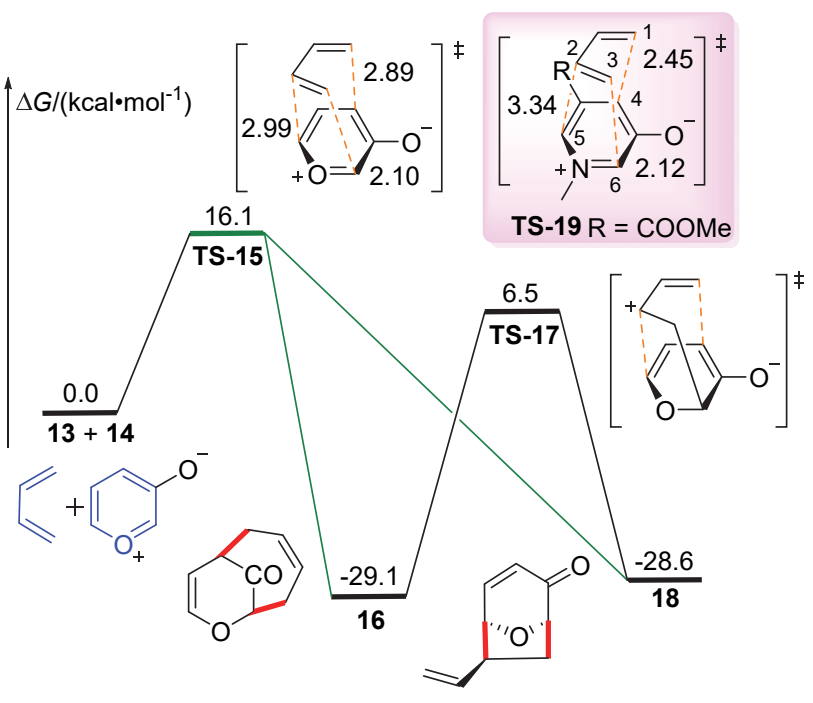

图 4 1,3-丁二烯和氧化吡啶鎓叶立德环加成反应势能面 Figure 4 Free energy profiles for cycloaddition of butadiene and oxido-pyrylium ylides

QM calculations were performed using M06-2X/6-311+ G**//SMD (DCM) level of theory. The bond lengths are in angstroms

成产物的现象，并认为酯基取代基的存在，抑制了反应 中过渡态的分叉行为. TS-19 存在较为极化的成键特征, 导致动态学模拟轨迹中, $92 \%$ 生成了 $[4+3]$ 环加成产物. 该研究不仅表明底物分子的取代基可影响分叉过渡态 的动态学性质, 而且证明了反应动态学能够预测传统势 能面无法解释的化学选择性问题.
分叉过渡态的选择性具有动态学特征, 有别于热力 学控制的产物或中间体, 且取代基的微小变化, 可能在 势能面上并不明显, 却可能导致明显的动态学影响. 2017 年, Houk 课题组 ${ }^{[29]}$ 报道了环庚三烯酮和 6,6-二甲 基-5-亚甲基-1,3-环戊二烯发生的 $[6+4]$ 环加成反应的反 应机制研究，如图 5 所示，作者利用 Progdyn 组件在 TS-22 附近的势能面区域启动准经典分子动态学模拟 (时间步长 $1 \mathrm{fs}$, 共 $500 \mathrm{fs}$ ), 计算结果表明, 两种环加成 产物 23 和 25 的比例为 $91 \%$ 和 $9 \%$, 这与产物的热力学 稳定性相反, 可能存在动态学控制. 作者还进一步研究 了甲基取代基对双周环分叉过渡态和产物分布的影响， 在反应物 $\gamma-\mathrm{C}$ 上连接甲基, 相应分叉过渡态的能量仅提 高了 $0.4 \mathrm{kcal} / \mathrm{mol}$, 轨迹结果中对应产物比例从原来的 $10 ： 1$ 提升到 $99 ： 1$, 说明甲基取代基导致的选择性差 别在动态学上可能更敏感, 这为高选择性环加成反应设 计提供了理论指导. 除此之外, 两种反应物在理论上有 多种环加成模式，作者利用过渡态理论成功定位了所有 的双周环分叉过渡态(图 $5, \mathrm{a} \sim \mathrm{d}$ ). 这些双周环分叉过渡 态都有三根部分形成的 $\mathrm{C}-\mathrm{C}$ 键, 其中一根 $\mathrm{C}-\mathrm{C}$ 键较 短为 $0.190 \mathrm{~nm}$ 左右, 而另外两根 $\mathrm{C}-\mathrm{C}$ 键键长约为 0.3 $\mathrm{nm}$, 这体现了双周环分叉过渡态的一般性特征，较短的 $\mathrm{C}-\mathrm{C}$ 键共享于两种分叉产物中, 较长的两根 $\mathrm{C}-\mathrm{C}$ 键则 是分叉产物所特有的键. 这种一般性的结构特征有助于 人们发现更多的过渡态分叉现象. 

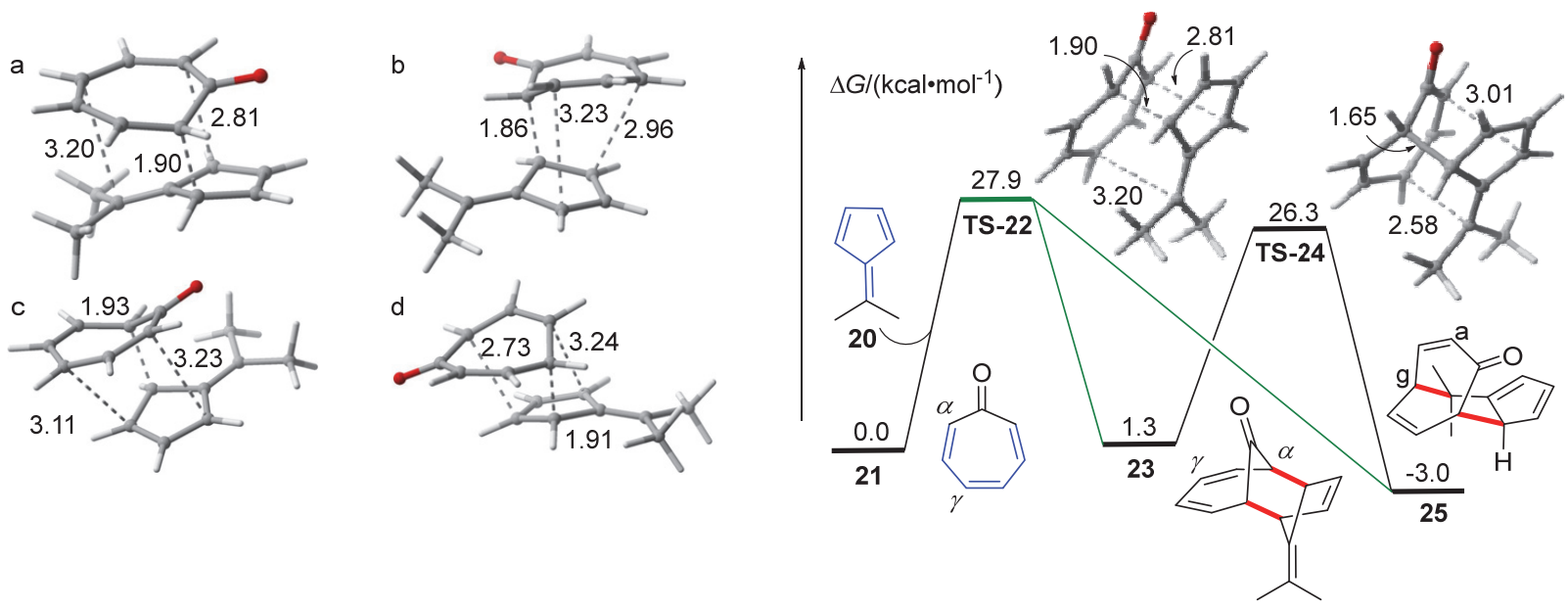

图 54 种双周环分叉过渡态和 $[6+4]$ 环加成反应势能面

Figure 5 Four bipericyclic transition states and free energy profiles for [6+4] cycloadditions

QM calculations were performed using PCM(diethyl ether)/M06-2X/ 6-311+G**//B3LYP-D3/6-31G* level of theory. The bond lengths are in angstroms

分叉过渡态现象也存在于复杂生物合成中, 而反应 动态学模拟也被人们用于发现重要的环加成过程. 2016 年, Houk 和 Singleton 等 ${ }^{[30]}$ 报道了 Spinosyn A 生物合成 新机制, 他们利用 Progdyn 组件进行动态学研究, 发现 该机制并不是形式上迪尔斯-阿尔德(Diels-Alder, D-A) 环加成, 而是双周环分叉过渡态产生的竞争性 $[6+4]$ 和 [4+2]环加成过程(图 6). 尽管实验观察到[4+2]环加成 产物 30 是唯一的产物, 但作者对 TS-27 附近势能面进行 动态学模拟(步长 $1 \mathrm{fs}$, 轨迹运动至 $28 、 30$ 或重新形成 26 停止)发现, [6+4]环加成产物 28 和[4+2]环加成产物 30 将以 $2.5: 1$ 的比例生成, 存在着明显竞争. 通过后过 渡态分叉 Cope 29-TS, [6+4]环加成产物 28 可较容易地
转化为 $[4+2]$ 环加成产物 30 , 活化能为 $20.7 \mathrm{kcal} / \mathrm{mol}$. 基于动态学的成键时间, 此体系从 TS-27 到形成环加成 产物 28 和 30 的中位数时间分别为 197 和 $171 \mathrm{fs}$, 两根 $\mathrm{C}-\mathrm{C}$ 键成键间隔中位数时间分别为 139 和 $173 \mathrm{fs}$, 故该 反应倾向于动态学分步成键过程 ${ }^{[31]}$. 虽然在多环协同 过程中, 化学家们往往更关注[4+2]环加成, 但此研究 说明 $[6+4]$ 环加成同样是不可忽略的重要机制.

而在 Heronamide A 生物合成中, 的确生成了更为 稳定的[6+4]环加成产物. 2015 年, Houk 课题组 ${ }^{[32]}$ 报道 了 Heronamide A生物合成中的双周环分叉导致的 $[6+4]$ 和[4+2]环加成反应. 随后, 2020 年, 余沛源和梁勇课题

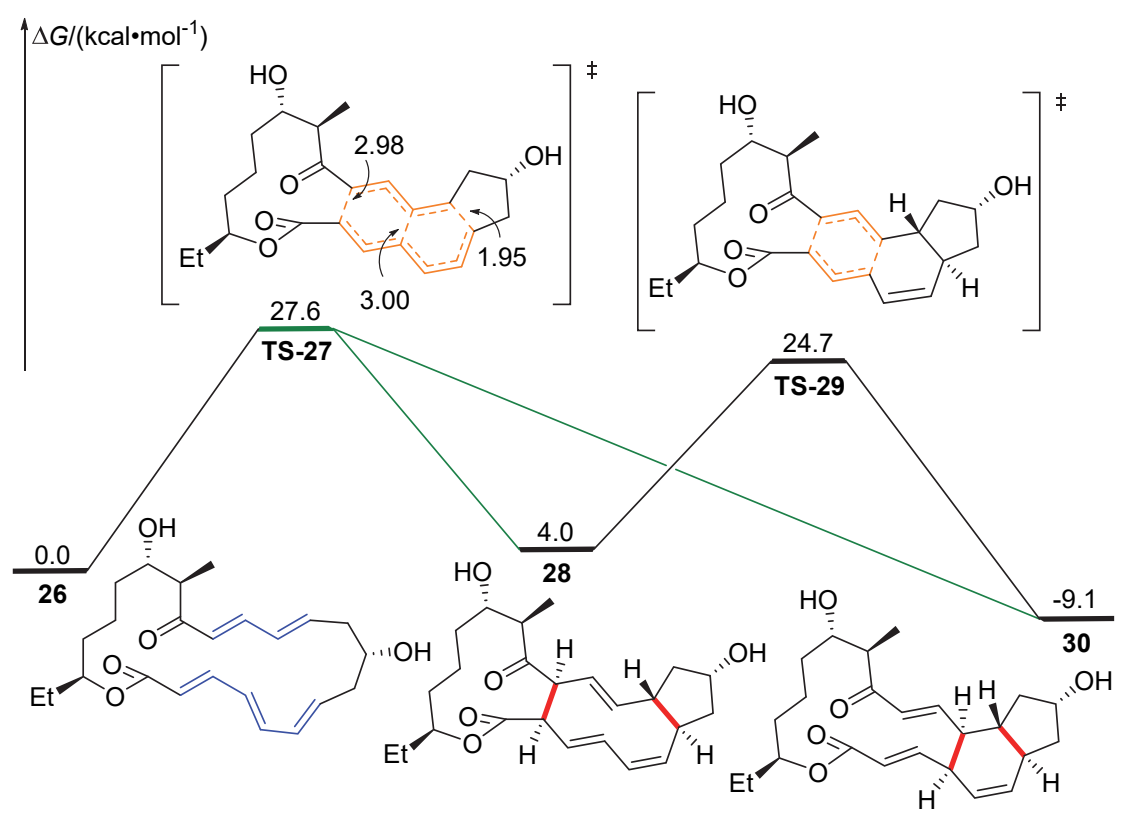

图 6 Spinosyn A 生物合成中的 $[6+4] 、[4+2]$ 环加成竞争反应势能面

Figure 6 Free energy profiles for competition of [6+4] and [4+2] cycloaddition in the biosynthesis of spinosyn A QM calculations were performed using SMD(H2O)/M06-2X/def2-TZVPP//B3LYP-D3(BJ)/6-31+G** level of theory. The bond lengths are in angstroms 
组 ${ }^{[33]}$ 联合报道了上述过程的动态学机制研究以及溶剂 影响，作者利用 Progdyn 组件进行气相模拟，用 AmberTools $16^{[34]}$ 进行显式水溶剂模型下的经典动力学 模拟. 如图 7 所示, 从 TS-32 启动轨迹(时间步长为 $1 \mathrm{fs,}$ 轨迹运动至生成 33、35 或重新形成 31 停止), 在气相和 显式水溶剂模型下, 生成的 $[6+4]$ 和 [4+2]环加成产物 33 和 35 的比例分别为 $16: 1$ 和 $56: 1$. 由于产物 33 的
偶极矩(4.31 D)远大于产物 35 的偶极矩(1.43 D), 作者推 断分叉过渡态的选择性受溶剂极性的影响较大，进一步 显示了生物合成中溶剂的重要性.

过渡态的分叉现象通常是导向两种产物或中间体 的双分叉类型，而多分叉过渡态的情况却极为少见. 2019 年, Houk 课题组 ${ }^{[35]}$ 报道了首例环加成反应过渡态 中存在的三周环分叉现象, 如图 8 所示, 作者利用 Prog-

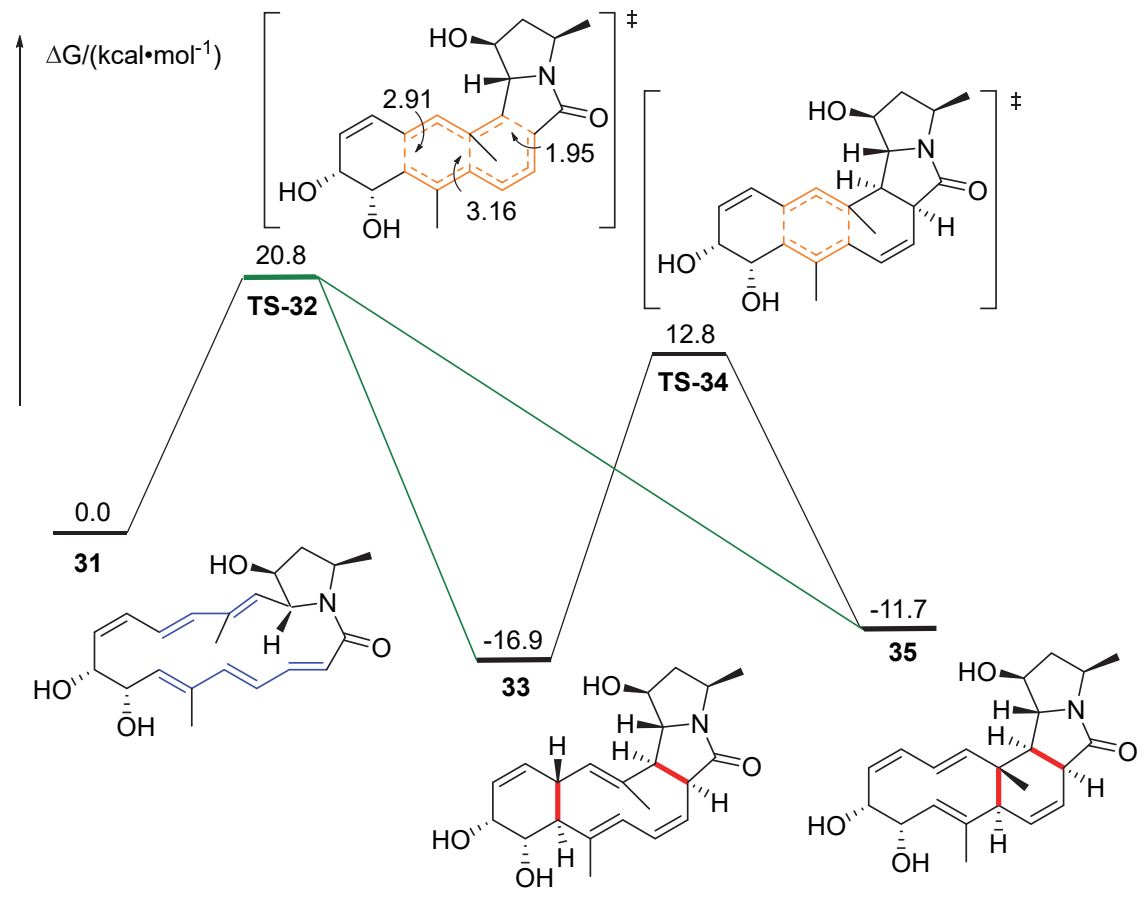

图 7 Heronamide A 生物合成中的 $[6+4] 、[4+2]$ 环加成反应势能面

Figure 7 Free energy profiles for $[6+4]$ and $[4+2]$ cycloadditions in biosynthesis of heronamide A

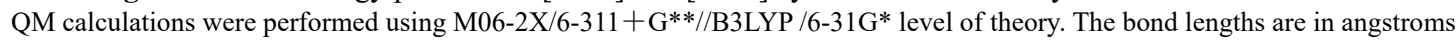

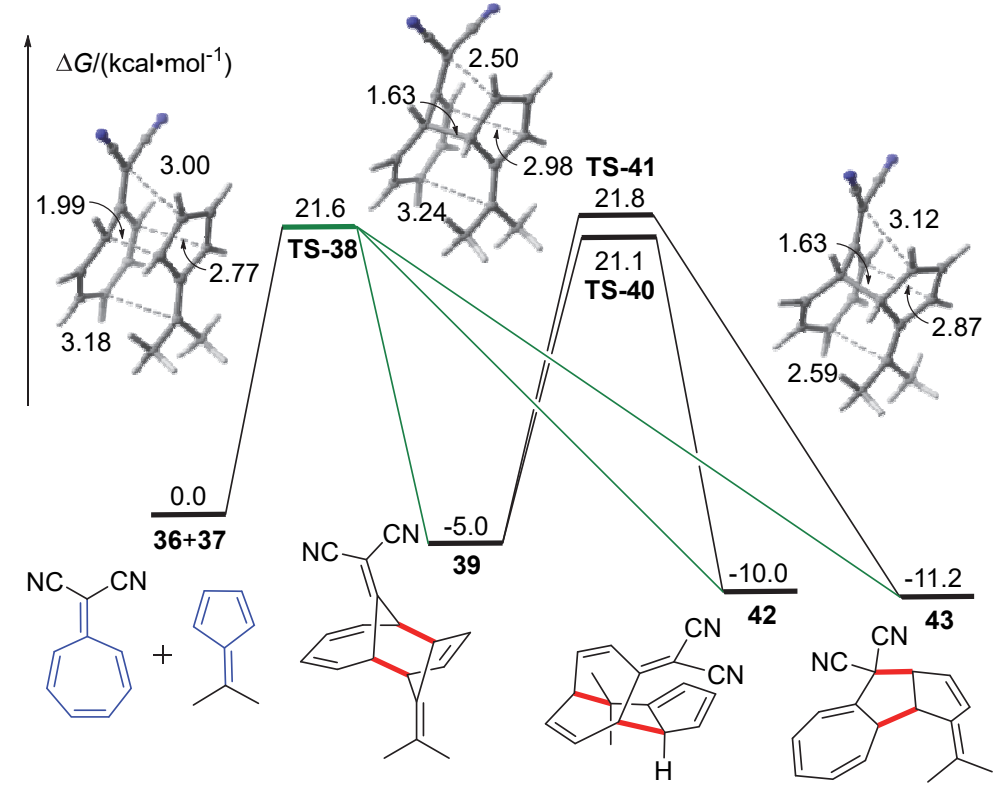

图 8 三分叉环加成反应势能面

Figure 8 Free energy profiles for tripericyclic cycloaddition

QM calculations were performed using $\omega \mathrm{B} 97 \mathrm{X}-\mathrm{D} / 6-31 \mathrm{G}^{*}$ level of theory. The bond lengths are in angstroms 
dyn 组件进行的轨迹模拟表明(时间步长 $1 \mathrm{fs}$, 共 $500 \mathrm{fs}$ ), 经过 TS-38 会生成 $[4+6]$ 、 $[6+4]$ 和 $[8+2]$ 三种环加成产 物 39, 42 和 43, 动态学预测三种产物的比例为 $25: 1$ : 1. 虽然与实验报道的主产物为 $[6+4]$ 和 $[8+2]$ 环加成产 物 42 和 43 并不一致, 但作者认为这是由于反应在经历 过渡态分叉后还会受到热力学控制. [4+6]环加成产物 39 在热力学上是最不稳定的, 它通过 Cope TS-40 和 TS-41 重排为 42 和 43. 关键分叉过渡态特征与双分叉时 类似, 最短的 $\mathrm{C}-\mathrm{C}$ 键首先决定了周环反应的方向, 其 次三种重要的次级轨道作用分别导向三种产物, 这个过 程由动态学控制, 而实验中观察到的产物是热力学控制 的结果.

\section{$1.2 \sigma$ 重排反应}

$\sigma$ 重排过程通常涉及到 $\sigma$ 键与多根键的生成和断裂, 形成环状过渡态, 也存在过渡态分叉的可能. 如图 9 所 示, 早期 Roth 等[36]通过实验研究了 1,2,6-庚三烯[3,3]- $\sigma$ 重排过程, 发现机制中存在双自由基中间体 46 和 47 , 且推断从反应物 44 到产物 49 的重排中约一半经历协同 机制. 随后, Borden 等的理论研究表明无法计算得到该 $\sigma$ 重排的过渡态, 因此很难解释实验中发现的机制 ${ }^{[37]}$. 于是, 2002 年, Carpenter 和 Borden 等 ${ }^{[38]}$ 报道了该 $\sigma$ 重排 过程的动态学研究, 发现了过渡态分叉现象. 作者利用 GAMESS 和一种半经验 MO 模型(AM1-SRP)进行动态 学轨迹计算, 结果表明, 从 TS-45 附近启动的轨迹中, $17 \%$ 直接生成了产物 49, 其余轨迹进入到势能面上双自 由基中间体 46、47 区域，这部分轨迹中有 $8 \%$ 在 $500 \mathrm{fs}$ 内离开此区域生成产物 49, 动态学轨迹定性地解释了 Roth 等的实验结果. 此研究表明, 能垒较高的双自由基 $\sigma$ 重排反应, 过渡态理论可能不适用, 反应能够动态地 生成必要的中间体和产物.

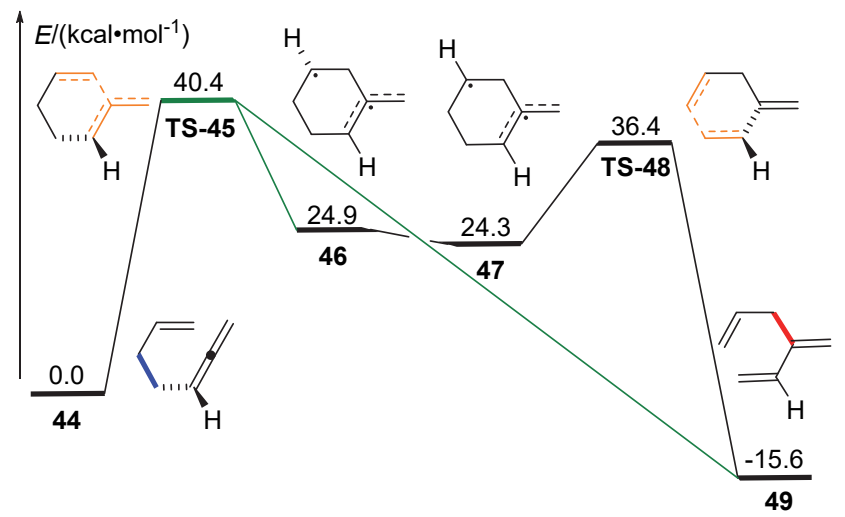

图 9 1,2,6-庚三烯重排势能面

Figure 9 Free energy profiles for 1,2,6-heptatriene rearrangement

QM calculations were performed using $\operatorname{CASSCF}(8,8) / 6-31 \mathrm{G}^{*}$ level of theory
2017 年, Silva 课题组 ${ }^{[39]}$ 报道了一例 $\sigma$ 重排反应中的 分叉现象, 卤素取代基高度影响了反应的选择性. 如 Scheme 1 所示, 反应物通过 $\sigma$ 重排经历两个结构相近过 渡态 TS-51 和 TS-52 进而发生过渡态分叉, 形成两种产 物 53 和 54. 如表 1 所示, 卤代后的 TS-51 能垒变化不大 $(>40 \mathrm{kcal} / \mathrm{mol})$, 但却极大地影响了反应动态学. 作者 利用 BOMD 从 TS-51 附近启动模拟(步长 $0.25 \mathrm{amu}^{1 / 2}$. bohr, 共 800 步), 研究发现对于氢取代 53 和 54 比例为 $1 ： 1$; 氟取代后得到了高选择性的结果, 产物 $53 \mathrm{~F}$ 和 $54 \mathrm{~F}$ 的比例达到了 $1: 10$, 这与过渡态 $\mathbf{T S} \mathbf{F}-\mathbf{5 1}$ 结构中 $\mathrm{C}(5)-\mathrm{C}(6)$ 键明显变短有关 (相较氢取代); $\mathbf{T S} \mathbf{C l}-51$ 启动 的轨迹仅产生 $53 \mathrm{Cl}$, 表明该过渡态在势能面上仅与 $53 \mathrm{Cl}$ 相连, 并且受过渡态结构中较长的 $C(5)-C(6)$ 键影响. 由于氟和氯对过渡态 $\mathrm{C}-\mathrm{C}$ 成键的作用不同, 可导向完 全相反的过渡态分叉选择性. 此工作中揭示了卤素取代 基的差异可调控动态学选择性, 加深了动态学调控选择 性的理解.

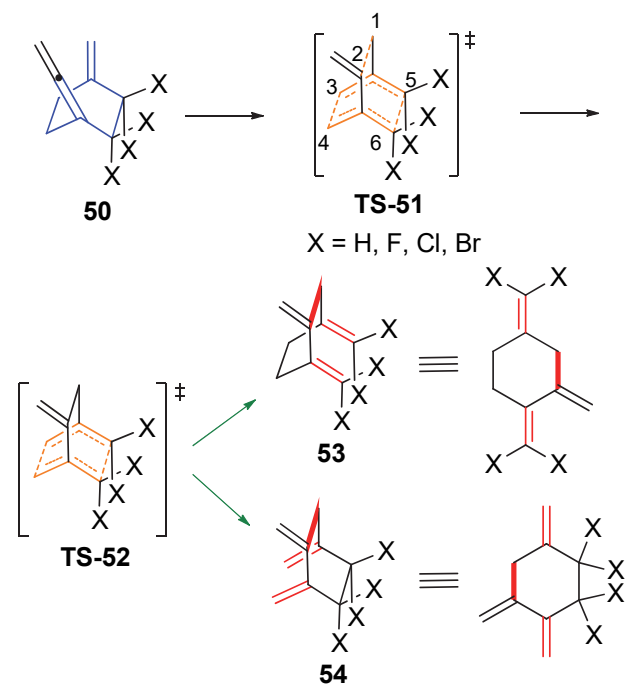

图式 1 两种 $[3,3] \sigma$-重排反应路径

Scheme 1 Two possible [3,3] б-rearrangement pathways

表 $1 \sigma$-重排中各中间体、过渡态及其卤代物自由能 ${ }^{a}$

Table 1 Free energies of intermediates, transition states and their halo-genated derivatives in $\sigma$-rearrangement

\begin{tabular}{|c|c|c|c|c|c|}
\hline Structure & $\Delta G$ & Structure & $\Delta G$ & Structure & $\Delta G$ \\
\hline 50 & 0.0 & $50 \mathrm{~F}$ & 0.0 & $50 \mathrm{Cl}$ & 0.0 \\
\hline TS-51 & 41.0 & $\mathrm{TS}_{\mathrm{F}}-51$ & 45.8 & $\mathbf{T S}_{\mathrm{Cl}}-51$ & 44.8 \\
\hline TS-52 & 36.9 & TSF-52 & 43.6 & $\mathbf{T S}_{\mathrm{Cl}-52}$ & 35.8 \\
\hline 53 & -11.5 & $\mathbf{5 3}_{\mathrm{F}}$ & -6.9 & $53 \mathrm{Cl}$ & -18.9 \\
\hline 54 & -11.5 & $54_{F}$ & -10.9 & $\mathbf{5 4}_{\mathrm{Cl}}$ & -10.7 \\
\hline
\end{tabular}

\section{3 碳正离子重排}

碳正离子作为重要的有机化学中间体之一, 认识其 反应机制并调控其化学选择性具有重要意义, 而碳正离 
子重排中也会出现过渡态分叉现象 ${ }^{[40]} .2011$ 年, Tantillo 和 Hase 等 ${ }^{[41]}$ 报道了一例松香酸生物合成中碳正离子重 排的势能面分叉现象, 如图 10 所示, 利用 Venus 组件从 TS-56 附近势能面区域启动的气相动态学模拟表明(时 间步长 $0.5 \mathrm{fs}$, 共 $1000 \mathrm{fs}$ ), 59\%轨迹在 100 300 fs 生成 了甲基迁移的中间体 57; 35\%轨迹在 120 650 fs 形成了 扩环的中间体 58, 而导向其他产物比例小于 5\%. 理论模 拟气相中无酶参与的动态学过程, 可得到比例可观的中 间体 58. 但在实验酶催化条件下只生成了中间体 57 而没 有任何的副产物, 作者认为这说明酶参与调控过渡态分 叉的选择性, 有望进一步研究此模型中酶的重要作用.

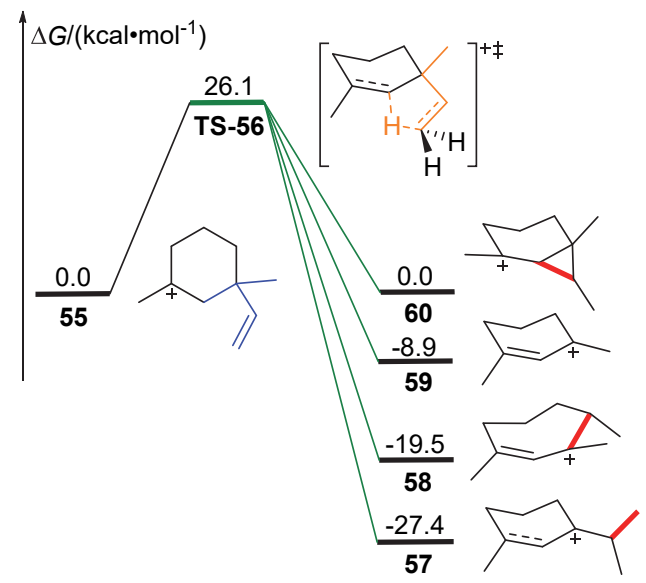

图 10 松香酸合成中碳正离子重排的势能面 Figure 10 Free energy profiles for a carbocation rearrangement in the synthesis of abietic acid

QM calculations were performed using B3LYP/6-31+ $\mathrm{G}^{* *}$ level of theory

2018 年, Tantillo 和 Sarpong 等 ${ }^{[42]}$ 报道了茴香酮型骨 架合成和动态学控制的机理研究, 如 Scheme 2 所示, 作 者利用 Progdyn 组件从 TS-62 附近启动的轨迹中(时间步 长 $1 \mathrm{fs}$, 共 $1000 \mathrm{fs}$ ), 66\%形成了中间体 63, 0.9\%形成了 中间体 64, 0.4\%在 TS-62 附近较平坦的势能面区域停留 了 $1000 \mathrm{fs}$ 以上, 其余轨迹回到了反应物. 此反应动态学 预测反应几乎完全转化为中间体 63 , 与实验结果一致.

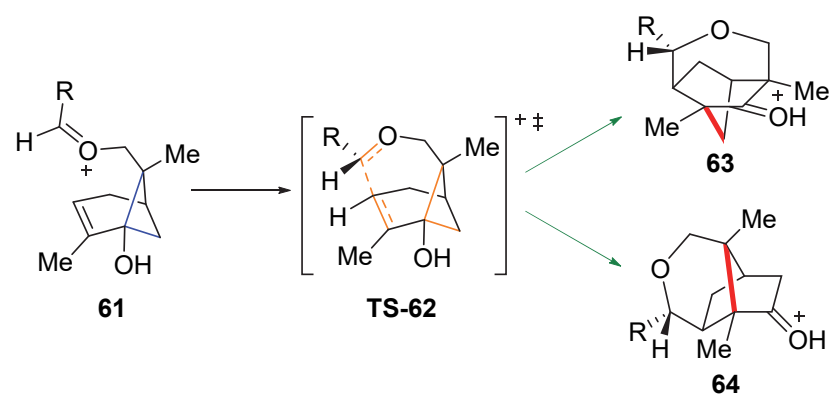

图式 2 Prins 重排中的两种反应路径

Scheme 2 Two pathways of Prins rearrangement
动态学模拟也表明较小的伯碳具有更活跃的运动状态, 其迁移能力较强进而主导了动态学的选择性.

\section{2 环加成协同-分步机制的动态学研究}

环加成过程(如卡宾环加成和 D-A 反应等)在实验和 理论上都有着广泛的研究, 许多实验证明该类反应经历 了立体专一的协同机制. 然而运用准经典分子动态学对 分子微观状态研究表明, 新形成的两根化学键在飞秒时 间尺度上并非同时形成，其成键时间间隔与反应机制关 系密切, 进而影响反应选择性. 因此, 准经典分子动态 学可用于判定协同一分步反应机制.

卡宾是有机反应中最为重要的中间体之一，其对烯 基的插入机制通常被认为是协同过程. 2011 年, Doubleday 和 Houk 等[43]报道了单重态 $\mathrm{CCl}_{2}$ 与 $\mathrm{CF}_{2}$ 和乙烯环 加成反应的动态机制. 尽管两类卡宾在反应势能面上区 别不大，但在反应过程中却展现了不同的动态学协同和 分步机制. 如 Scheme 3 所示, 利用 Venus 组件 UB3LYP 计算 $\mathbf{R} 1$ 和 $\mathbf{R 2}$ 反应以及 $\mathrm{mUB} 3 \mathrm{LYP}(12 \% \mathrm{HF}$ 成分)计算 $\mathbf{R 2}$ 的动态学模拟中发现(时间步长 $1 \mathrm{fs}$, 轨迹运动至生 成产物或回到反应物), $\mathrm{CCl}_{2}$ 与 $\mathrm{CF}_{2}$ 和乙烯的加成反应有 三个共同特征: (1)两根 $\mathrm{C}-\mathrm{C}$ 键的形成有先后顺序; (2) 双自由基 $\mathrm{CCCX}_{2}$ 基团转变到允许轨道重叠时才会形成 第二根 $\mathrm{C}-\mathrm{C}$ 键 $(\mathbf{R} 3$ 模式); (3)烯基 $\mathrm{C}-\mathrm{C}$ 键不会发生旋 转. 而不同的是, 卡宾 $\mathrm{CCl}_{2}$ 体系在第一根 $\mathrm{C}-\mathrm{C}$ 成键 65 fs 之后有 95\%完成了环加成过程, 表现为动态学协同过 程; 而卡宾 $\mathrm{CF}_{2}$ 受计算方法 $\mathrm{HF}$ 成分影响较大, 采用 mUB3LYP 可以得到近似 $\mathrm{CCl}_{2}$ 动态学协同过程; 而原 UB3LYP 产生了较难转化的双自由基中间体，双自由基 所需的翻转势垒 $(\approx 6 \mathrm{kcal} / \mathrm{mol})$ 有效地延长了双自由基 的寿命, 形成动态学分步过程. 该研究反应了不同卤素 取代基对卡宾环加成机制的影响，显示了动态学研究在 区分环加成协同和分步机制中的特点.

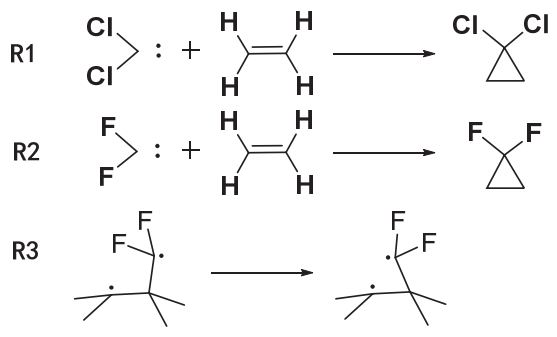

图式 $3 \mathrm{CCl}_{2}$ 和 $\mathrm{CF}_{2}$ 与乙烯的反应以及双自由基基团的翻转 Scheme 3 Reactions of $\mathrm{CCl}_{2}$ and $\mathrm{CF}_{2}$ with ethylene and the inversion of diradical complex

QM calculations were performed using UB3LYP/6-31G* and mUB3LYP/ 6-31G* (HF exchange in UB3LYP was reduced from $20 \%$ to $12 \%$, and the contribution of local exchange was adjusted to $88 \%$ ) level of theory

2012 年, Doubleday 和 Houk 等[31]报道了 8 种 D-A 
反应在动态学、过渡态和成键时间尺度上的反应机制研 究. 如 Scheme 4 所示, R4-9 对应的鞍点结构是完全对称 的，受动态学影响，这些反应均倾向于协同反应机制但 存在异步性; 对于非对称反应 R10 和 R11, 受反应的静 态结构不对称和动态效应的共同影响, 异步性提升, 更 倾向于分步机制. 动态学模拟表明(298 K 下从 $\mathbf{R 4} \sim \mathbf{R 9}$ 对应过渡态启动的动态学轨迹步长为 $0.12 \mathrm{amu}^{1 / 2} \bullet \mathrm{bohr}$, 298 K 下 R10, R11 和 $1180 \mathrm{~K}$ 下 $\mathbf{R 4}$ 对应动态学步长为 $0.25 \mathrm{amu}^{\frac{1}{2} / 2}$ bohr), $\mathbf{R 4} \sim \mathbf{R 9}$ 对应的轨迹结果较为相似, 展 现了 $[4+2]$ 环加成反应的常见的协同特征, 两根 $\mathrm{C}-\mathrm{C}$ 键成键平均时间间隔 $5 \mathrm{fs}$, 远小于 $\mathrm{C}-\mathrm{C}$ 键伸缩振动周 期(30 $60 \mathrm{fs})$. 基于此, 作者定义了成键 $[d(\mathrm{C}-\mathrm{C}) \leqslant 0.17$ $\mathrm{nm}$ ]时间差 $<60 \mathrm{fs}$ 为动态学协同过程, 而 $\geqslant 60 \mathrm{fs}$ 则为动 态学分步过程. R10 和 R11 比 R4-9 具有更强的异步性,

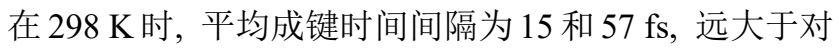
称反应的平均成键时间间隔, 但大多数轨迹依然是动态 协同的. 作者进一步研究 $1180 \mathrm{~K}$ 的 R4 和 $298 \mathrm{~K}$ 的 R10 和 R11 对应轨迹发现, 体系形成了寿命约 $1000 \mathrm{fs}$ 的双 自由基中间体，进而通过动态分步的方式生成环加成产 物, 说明升高温度可以提高 $\mathrm{D}-\mathrm{A}$ 反应的动态学分步机制 比例. 此研究定义了协同一分步机制的动态学标准, 为 后续研究提供坚实的理论基础.

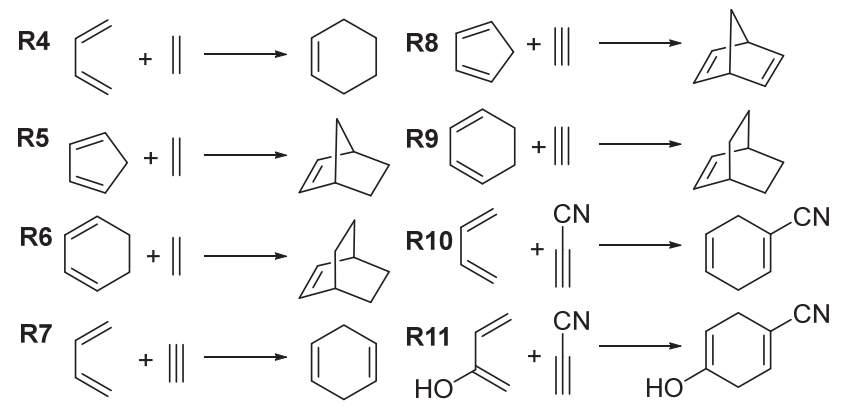

图式 46 类对称和 2 类不对称 D-A 反应

Scheme 4 Six types of symmetrical and two types of asymmetric D-A reactions

QM calculations were performed using UB3LYP/6-31G* level of theory

2016 年, Houk 课题组 ${ }^{[44]}$ 报道了 Dehydro-Diels-Alder (DDA)反应的动态学研究, 如 Scheme 5 所示, 6 种反应 协同机制下的反应活化能有着显著差异, 而分步过程的 反应活化能却大致相同. 对 R17 的动态学模拟表明(时 间步长 $1 \mathrm{fs}$, 共 $500 \mathrm{fs}$ ), 协同机制下丁二炔在到达过渡 态之前经历了大幅度弯曲振动, 这与线性的丁二炔形成 过渡态结构需要较大形变有关; 而其分步机制中分子弯 曲振荡程度较小，在 150 条的分步机制轨迹模拟中没有 观察到最终成环产物. 这是由于双自由基中间体的稳定 性高于生成第二根 $\mathrm{C}-\mathrm{C}$ 键的过渡态, 导致成环过程需 要皮秒级的时间来完成 $\mathrm{C}-\mathrm{C}$ 键旋转和振动激发. 该研
究表明底物分子形成过渡态所需的扭转能对协同机制 起决定性作用，而随着协同机制中扭转能提升，分步机 制会变得更有竞争力.

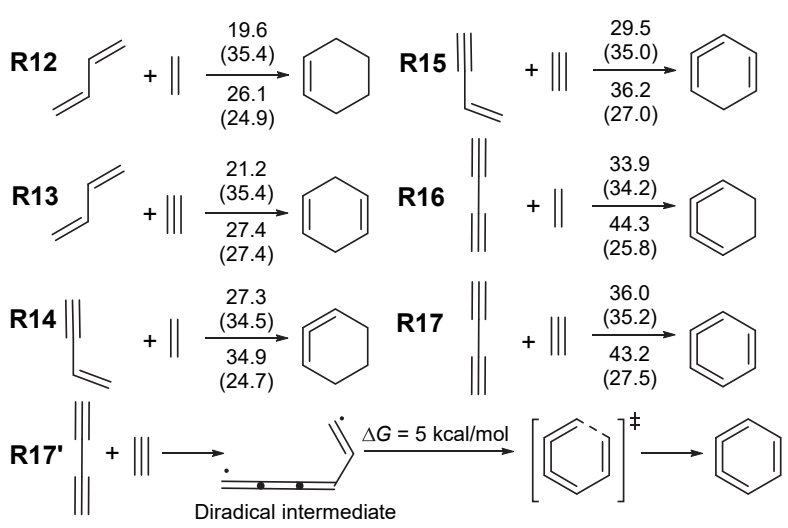

图式 $5 \mathrm{R} 12, \mathbf{R} 13$ 的一般 D-A 反应和 R14 $\sim \mathrm{R} 17$ 的 DDA 反应 (R17'展示了 R17 的双自由基机制)

Scheme 5 General D-A reactions of R12, R13, and DDA reactions of R14 $\sim \mathbf{R 1 7}$ (R17' shows the diradical mechanism of R17) The electronic energies above and below the arrows are the activation energy and distorsion energy of the reaction under the concerted mechanism (the corresponding electronic energy in the stepwise mechanism are given in brackets). QM calculations were performed using (U)M06$2 \mathrm{X} / 6-311+\mathrm{G}^{* *}$ level of theory. Energies are given in $\mathrm{kcal} / \mathrm{mol}$

2020 年, 钟龙华课题组 ${ }^{[45]}$ 通过 DFT 计算和反应动 态学研究了铁催化 oxa-Diels-Alder (ODA)反应的机制. 如图 11 所示, 作者利用 Progdyn 组件从 TS-67 附近启动 动态学模拟(轨迹计算至 $\mathrm{C}-\mathrm{C}$ 或 $\mathrm{C}-\mathrm{O}$ 成键, 或反应物 远离至 $>0.33 \mathrm{~nm}$ 停止). 如表 2 所示，在不同条件下，铁 催化的成键时间和成键时间间隔都明显大于无金属催

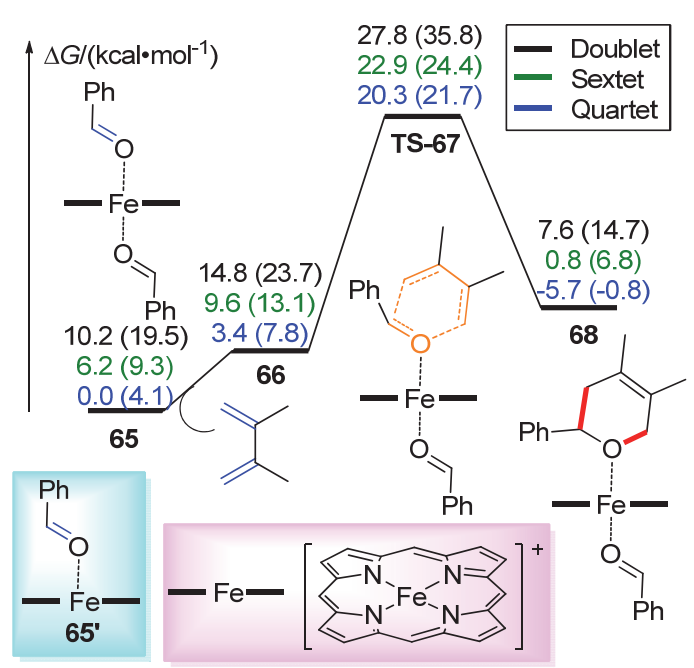

图 11 铁催化 ODA 反应势能图

Figure 11 Free energy profiles for Fe-catalyzed ODA reaction The corresponding free energies of the five-coordinated Fe-catalyzed structures (such as $\mathbf{6 5}^{\prime}$ ) is indicated in brackets. QM calculations were performed using SMD/B3LYP-D3/6-31G*(def2-TZVP for Fe) level of theory 
化体系, 而沿 $\mathrm{Fe}-\mathrm{O}$ 键轴方向施加 $-0.0030 \mathrm{au}$ 的外加电 场(Oriented External Electric Field, OEEF), 会进一步增 加分步过程比例, 甚至导致五配位六重态体系趋近于完 全的分步过程 $(96 \%)$. 这是首次发现 D-A 反应动态学协 同一分步机制受催化剂金属中心配位模式、自旋状态和 OEEF 影响, 丰富了我们对 ODA 反应动态学协同-分步 机制的认识, 同时帮助化学家们设计合成更多具有挑战 性的 ODA 反应.

表 2 准经典分子动态学轨迹数量

Table 2 Number of the trajectories

\begin{tabular}{|c|c|c|c|}
\hline & Gas & Solution & Solution $+\mathrm{OEEF}$ \\
\hline Uncatalyzed & $100\left(0^{c}\right)\left[5^{d}\right]$ & $100\left(0^{c}\right)\left[6^{d}\right]$ & - \\
\hline $6-\mathrm{c}^{4} \mathrm{Fe}^{a}$ & $100\left(21^{c}\right)\left[40^{d}\right]$ & $130\left(26^{c}\right)\left[50^{d}\right]$ & $130\left(86^{c}\right)\left[174^{d}\right]$ \\
\hline $5-c^{4} \mathrm{Fe}^{b}$ & $130\left(39^{c}\right)\left[59^{d}\right]$ & $130\left(54^{c}\right)\left[98^{d}\right]$ & $130\left(88^{c}\right)\left[235^{d}\right]$ \\
\hline $5-c^{6} \mathrm{Fe}^{b}$ & $100\left(74^{c}\right)\left[103^{d}\right]$ & $130\left(87^{c}\right)\left[199^{d}\right]$ & $130\left(96^{c}\right)\left[414^{d}\right]$ \\
\hline
\end{tabular}

\section{3 “火山口” 型势能面的动态学效应}

“火山口” 型势能面如图 12 所示, 当相邻的两个过 渡态(TS-70 和 TS-72)和中间体(71)能量差异较小, 势能 面形成类似于 “火山口” 的形状的势能面. 人们研究发 现该势能面下的化学反应可能会受动态学效应影响, 以 接近协同的方式快速通过这一势能面区域. 这些现象很 难用传统过渡态理论中活化能的相对高低来解释, 而准
经典分子动态学提供了理解机理本质的研究手段.

2018 年, Ess 课题组 ${ }^{[21]}$ 报道了一例 Ir 催化负氢转移 的反应动态学机制，发现该机制有可能绕过重要的高价 Ir-H 中间体. 基于 DFT 计算的反应势能面如图 12 所示, 利用 DynSuite 组件在 TS-70 附近启动动态学轨迹计算 甲烷分子的 $\mathrm{C}-\mathrm{H}$ 断裂和负氢转移过程(时间步长 $1 \mathrm{fs}$, 共 500 $800 \mathrm{fs}$ ), 其中代表性轨迹在 $75 \mathrm{fs}$ 之后就形成了 新的 C- $\mathrm{H}$ 键, 这表明在势能面上 TS-70 与 TS-72 发生 了偶合，最终在 $375 \mathrm{fs}$ 时甲烷完全离解(与 Ir 金属中心的 距离超过了 $0.35 \mathrm{~nm}$ ), 整个反应过程绕过了中间体 71 和 73 , 这类轨迹占全部轨迹的 $11 \%$. 而沿着最低能量路径 得到 Ir-H 中间体和回到反应物的比例分别为 $81 \%$ 和 $8 \%$. 作者还计算了苯分子 $\mathrm{C}-\mathrm{H}$ 的断裂和负氢转移过程，从 TS-75 附近区域启动的动态学轨迹, 与 TS-70 类似, 轨 迹中也出现了 $18 \%$ 的绕过 Ir-H 中间体的轨迹, 形成 Ir-H 中间体和回到反应物的比例分别为 $63 \%$ 和 $19 \%$. 这些轨 迹生成的甲烷分子由于自身的运动加速了其离解. 此研 究表明, 部分轨迹能够绕过势能面上的重要的中间体, 只需一步类似协同的过程生成了产物，这丰富了我们对 传统势能面的认识.

2020 年, 郑超课题组 ${ }^{[46]}$ 对 $\mathrm{Cp} \mathrm{p}^{*} \mathrm{M}$ (III) $(\mathrm{M}=\mathrm{Co}, \mathrm{Rh}$, Ir)类配合物中 1,4-负氢迁移中的 DFT 计算和动态学效 应进行了研究, 发现分步反应过程中的活性中间体稳定 性逐步下降(从 Ir 到 $\mathrm{Co}$ ), 特别是 $\mathrm{Cp} \mathrm{p}^{*} \mathrm{Co}(\mathrm{III})$ 中, 中间体
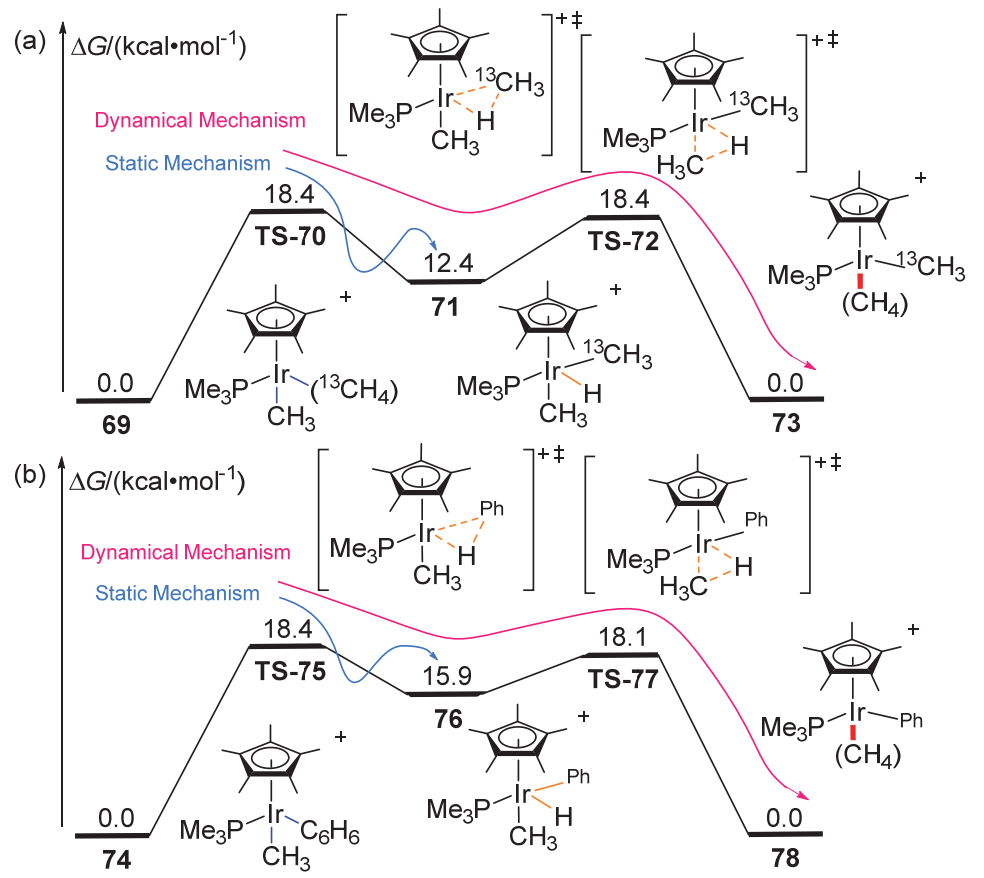

图 12 (a) ${ }^{13} \mathrm{CH}_{4}$ 和(b)苯的 $\mathrm{C}-\mathrm{H}$ 活化势能面

Figure 12 Free energy profiles for $\mathrm{C}-\mathrm{H}$ activation of (a) ${ }^{13} \mathrm{CH}_{4}$ and (b) benzene QM calculations were performed using M06/6-31G*[LANL2DZ for Ir] level of theory 
能量与过渡态能量十分接近, 这表明反应可能存在动态 学效应. 随后, 作者以 $\mathrm{Co} 、 \mathrm{Rh}$ 催化体系启动准经典分 子动态学研究 (以 $1 \mathrm{fs}$ 为步长, 最长 $1.5 \mathrm{ps}$, 每组 40 条轨 迹), 计算结果如图 13 所示, $\mathrm{Rh}$ 催化受动态学效应影响 形成动态学分步过程, 整体轨迹持续时间较长 $(>48 \mathrm{fs})$; Co 催化则更倾向于动态学协同的过程, 且多数轨迹在 $60 \mathrm{fs}$ 内完成了 1,4-负氢迁移过程. 该研究证明了金属中 心可以调控反应动态学机制, 并加深了人们对负氢迁移 过程中协同-分步机制的理解.

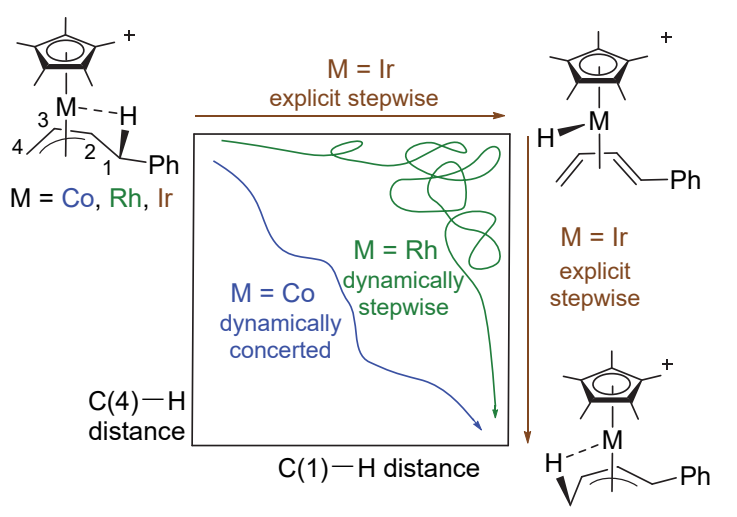

图 $13 \mathrm{Cp} * \mathrm{M}(\mathrm{III})(\mathrm{M}=\mathrm{Co}, \mathrm{Rh}, \mathrm{Ir})$ 催化的 1,4-负氢迁移机理 More O'Ferrall-Jencks 图

Figure 13 A More O'Ferrall-Jencks-type diagram for the 1,4hydride transfer mechanism mediated by $\mathrm{Cp} * \mathrm{M}(\mathrm{III})(\mathrm{M}=\mathrm{Co}, \mathrm{Rh}$, Ir)

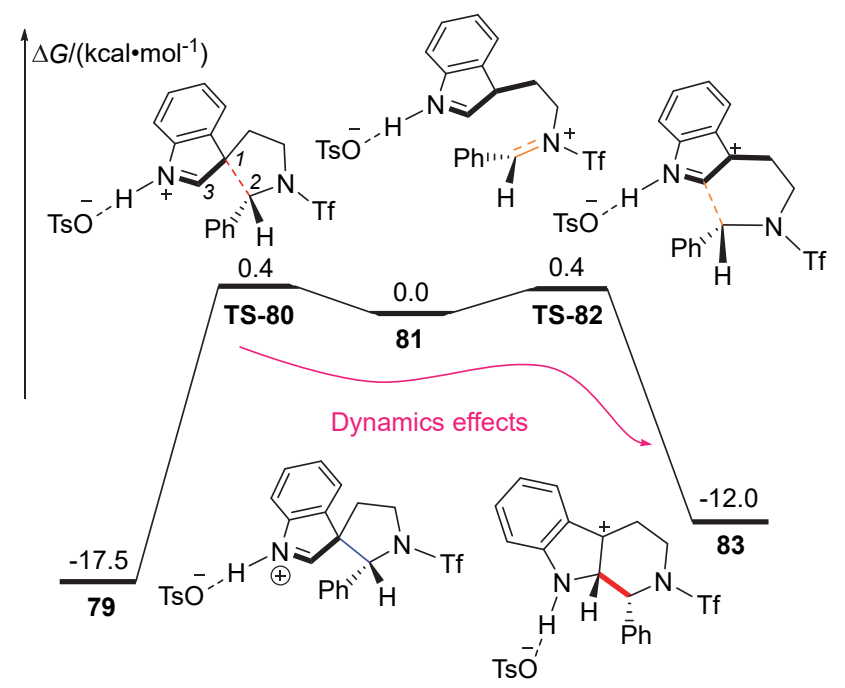

图 14 Pictet-Spengler 反应势能面

Figure 14 Free energy profiles for Pictet-Spengler reaction QM calculations were performed using PBE1PBE/6-311+ $\mathrm{G}^{* *}$ level of theory

2018 年, 游书力课题组 ${ }^{[47]}$ 报道了 Pictet-Spengler 反 应的机制和实验研究. 如图 14 所示, TS-80、TS-82 和中 间体 81 的能量非常接近, 呈现出类似于 “火山口” 形状 的势能面. 作者利用 BOMD 来评估反应中的动态学效
应(轨迹计算至 $\mathrm{C}(1)-\mathrm{C}(2)$ 或 $\mathrm{C}(2)-\mathrm{C}(3)$ 成键，或算至 2 $\mathrm{ps}$ 的总时间停止), 从 TS-80 开始的 100 条轨迹可被分为 5 类: (1)从中间体 79 到 83 (44 条); (2) C(1)- $\mathrm{C}(2)$ 键断裂 后重新成键形成中间体 79 (44 条); (3)在中间体 81 区域 附近震荡 $(8$ 条); (4)经过中间体 83 又回到 TS-80, 随后又 重新形成中间体 83 (3 条); (5) 1 条轨迹完全离开了势能 面上的平坦区域, 亚胺基和吲哚环的距离超过了 0.4 $\mathrm{nm}$. 其中, 第一类轨迹持续的平均时间为 $755 \mathrm{fs}$, 比一 般的动态学协同过程寿命长一个数量级. 这类轨迹提供 了一条直接从螺环中间体 79 生成并环中间体 83 的反应 路径, 说明二者之间存在着动态学关联. 这种动态学效 应保证了反应的立体化学, 大多数的轨迹都会持续震荡 数百飞秒的时间, 但亚胺基和吲哚环之间的距离基本保 持在 $0.35 \mathrm{~nm}$ 之内, 确保产物的立体化学不会发生改变. 此研究提供了对 Pictet-Spengler 反应机制的新理解, 为 合成螺环吲哚胺类化合物提供理论指导.

\section{4 总结与展望}

介绍了准经典分子动态学在分叉过渡态的选择性、 动态学协同-分步机制和 “火山口” 型势能面的动态学 效应等方面的重要应用, 从中可看出, 在形成过渡态的 关键成/断键区域, 化学键结构较为类似时, 很可能需要 考虑准经典分子动态学来进行研究. 分叉过渡态中, 当 分叉中间体之间的转化速度较慢时, 准经典分子动态学 影响较大. 此时, 过渡态分叉过程受势能面形状和动态 学控制, 轨迹结果的统计学分布可预测分叉过渡态的选 择性. 传统上被认为是协同过程的环加成反应, 两根新 的化学键并不会完全同时生成. 受环加成过渡态结构对 称性下降或环境温度升高等条件影响，反应异步性可能 会增强, 需考虑准经典分子动态学来研究其飞秒级时间 尺度的成键过程，可定量区分反应的协同-分步竞争机 制. 当反应出现 “火山口” 型势能面时, 会有明显的动 态学效应. 此时反应机制既有可能会绕过其势能面上的 中间体, 以接近动态学协同的过程来推动化学反应的进 行, 也可能出现长寿命中间体而表现为动态学分步效 应，这拓宽了我们对传统化学反应机制的认知. 此外, Hase 等 ${ }^{[48-49]}$ 还对分子碰撞的基元反应机制的分子动态 学进行深入的研究, 例如, 相较于传统简单的 $\mathrm{S}_{\mathrm{N}} 2$ 机制 描述, 动态学揭示了不同碰撞能量下反应分子直接剥 离、反弹、侧面进攻或间接迂回、形成离子偶极复合物 等详细的机制过程.

准经典分子动态学应用于反应机制研究的例子还 比较有限，存在广阔的发展空间，有望拓展到更多体系 的微观过程, 例如复杂多变的构象转化所引发的机制变 化, 进而探索微小扰动下, 反应的微观过程; 动态氢键 
在化学反应中的形成和转化, 进而探索催化过程和生命 体系中化学过程的动态效应等, 这为反应机制的理解提 供了新的可能. 尽管准经典分子动态学在揭示有机化学 反应机制中发挥着独特的作用, 但其应用于复杂体系的 研究还较少, 部分原因是由于计算效率不高. 比如, 动 态学模拟一般只涉及到相邻一两个中间体, 受中间体所 在的势井的束缚, 轨迹运动范围有限, 需要提高计算速 度才有可能使轨迹连接到结构差异较大的中间体. 再 者, 准经典分子动态学模拟大多处于皮秒级别的计算水 平, 提升计算效率, 模拟更长时间的化学微观过程, 对 深入研究宏观上通常以分钟到小时为时间单位的化学 反应本身具有重要意义, 这可能需要多学科交叉融合, 包括数理以及计算机软硬件等领域研究人员的共同努 力才能够实现.

\section{References}

[1] Blais, N. C.; Bunker, D. L. J. Chem. Phys. 1962, 37, 2713.

[2] Bunker, D. L. J. Chem. Phys. 1964, 40, 1946.

[3] Bunker, D. L. J. Chem. Phys. 1962, 37, 393.

[4] Hohenberg, P.; Kohn, W. Phys. Rev. 1964, 136, 864.

[5] Kohn, W.; Sham, L. J. Phys. Rev. 1965, 140, 1133.

[6] Zhang, D. H.; Collins, M. A.; Lee, S.-Y. Science 2000, 290, 961.

[7] Xie, Y.; Zhao, H.; Wang, Y.; Huang, Y.; Wang, T.; Xu, X.; Xiao, C.; Sun, Z.; Zhang, D. H.; Yang, X. Science 2020, 368, 767.

[8] Paranjothy, M.; Sun, R.; Zhuang, Y.; Hase, W. L. Comput. Mol. Sci. 2013, 3, 296.

[9] Pratihar, S.; Ma, X.; Homayoon, Z.; Barnes, G. L.; Hase, W. L. J. Am. Chem. Soc. 2017, 139, 3570.

[10] Verlet, L. Phys. Rev. 1967, 159, 98.

[11] Hollingsworth, S. A.; Dror, R. O. Neurone 2018, 99, 1129.

[12] Car, R.; Parrinello, M. Phys. Rev. Lett. 1985, 55, 2471.

[13] Helgaker, T.; Uggerud, E.; Jensen, H. J. A. Chem. Phys. Lett. 1990, $173,145$.

[14] Uggerud, E.; Helgaker, T. J. Am. Chem. Soc. 1992, 114, 4265.

[15] Barnett, R. N.; Landman, U. Phys. Rev. B 1993, 48, 2081.

[16] Hase, W. L.; Duchovic, R. J.; Hu, X.; Komornicki, A.; Lim, K.; Lu, D.-H.; Peslherbe, G. H.; Swamy, K. N.; Vande Linde, S. R.; Wang, H.; Wolfe, R. J. Quant. Chem. Prog. Ex. 1996, 16, 671

[17] Li, G.; Hase, W. L. J. Am. Chem. Soc. 1999, 121, 7124

[18] Xie, J.; Sun, R.; Siebert, M. R.; Otto, R.; Wester, R.; Hase, W. L. J. Phys. Chem. A 2013, 117, 7162.

[19] Peslherbe, G. H.; Hase, W. L. J. Chem. Phys. 1996, 104, 7882.

[20] Singleton, D. A.; Hang, C.; Szymanski, M. J.; Greenwald, E. E. J. Am. Chem. Soc. 2003, 125, 1176.

[21] Carlsen, R.; Wohlgemuth, N.; Carlson, L.; Ess, D. H. J. Am. Chem. Soc. 2018, 140, 11039.
[22] Schmidt, M. W.; Baldridge, K. K.; Boatz, J. A.; Elbert, S. T.; Gordon, M. S.; Jensen, J. H.; Koseki, S.; Matsunaga, N.; Nguyen, K. A.; Su, S.; Windus, T. L.; Dupuis, M.; Montgomery Jr, J. A. J. Comput. Chem. 1993, 14, 1347.

[23] Ess, D. H.; Wheeler, S. E.; Iafe, R. G.; Xu, L.; Çelebi-Ölçüm, N.; Houk, K. N. Angew. Chem., Int. Ed. 2008, 47, 7592.

[24] Hare, S. R.; Tantillo, D. J. Pure Appl. Chem. 2017, 89, 679.

[25] Caramella, P.; Quadrelli, P.; Toma, L. J. Am. Chem. Soc. 2002, 124, 1130.

[26] Zhang, L.; Wang, Y.; Yao, Z.-J.; Wang, S.-Z.; Yu, Z.-X. J. Am. Chem. Soc. 2015, 137, 13290.

[27] Burns, J. M.; Boittier, E. D. J. Org. Chem. 2019, 84, 5997.

[28] Fu, C.; Lora, N.; Kirchhoefer, P. L.; Lee, D. R.; Altenhofer, E.; Barnes, C. L.; Hungerford, N. L.; Krenske, E. H.; Harmata, M. Angew. Chem., Int. Ed. 2017, 56, 14682.

[29] Yu, P.; Chen, T. Q.; Yang, Z.; He, C. Q.; Patel, A.; Lam, Y.-H.; Liu, C.-Y.; Houk, K. N. J. Am. Chem. Soc. 2017, 139, 8251.

[30] Patel, A.; Chen, Z.; Yang, Z.; Gutiérrez, O.; Liu, H.-W.; Houk, K. N.; Singleton, D. A. J. Am. Chem. Soc. 2016, 138, 3631.

[31] Black, K.; Liu, P.; Xu, L.; Doubleday, C.; Houk, K. N. Proc. Natl. Acad. Sci. U. S. A. 2012, 109, 12860.

[32] Yu, P.; Patel, A.; Houk, K. N. J. Am. Chem. Soc. 2015, 137, 13518.

[33] Zhang, C.; Wang, X.; Chen, Y.; He, Z.; Yu, P.; Liang, Y. J. Org. Chem. 2020, 85, 9440 .

[34] Salomon-Ferrer, R.; Case, D. A.; Walker, R. C. Comput. Mol. Sci. 2013, 3, 198

[35] Xue, X.-S.; Jamieson, C. S.; Garcia-Borràs, M.; Dong, X.; Yang, Z.; Houk, K. N. J. Am. Chem. Soc. 2019, 141, 1217.

[36] Roth, W. R.; Wollweber, D.; Offerhaus, R.; Rekowski, V.; Lennartz, H.-W.; Sustmann, R.; Müller, W. Chem. Ber. 1993, 126, 2701.

[37] Hrovat, D. A.; Duncan, J. A.; Borden, W. T. J. Am. Chem. Soc. 1999, 121,169 .

[38] Debbert, S. L.; Carpenter, B. K.; Hrovat, D. A.; Borden, W. T. J Am. Chem. Soc. 2002, 124, 7896

[39] Villar López, R.; Faza, O. N.; Silva López, C. J. Org. Chem. 2017, $82,4758$.

[40] Hare, S. R.; Tantillo, D. J. Beilstein J. Org. Chem. 2016, 12, 377.

[41] Siebert, M. R.; Zhang, J.; Addepalli, S. V.; Tantillo, D. J.; Hase, W. L. J. Am. Chem. Soc. 2011, 133, 8335.

[42] Blümel, M.; Nagasawa, S.; Blackford, K.; Hare, S. R.; Tantillo, D. J.; Sarpong, R. J. Am. Chem. Soc. 2018, 140, 9291.

[43] Xu, L.; Doubleday, C. E.; Houk, K. N. J. Am. Chem. Soc. 2011, 133, 17848

[44] Yu, P.; Yang, Z.; Liang, Y.; Hong, X.; Li, Y.; Houk, K. N. J. Am. Chem. Soc. 2016, 138, 8247.

[45] Yang, Y.; Zhang, X.; Zhong, L.-P.; Lan, J.; Li, X.; Li, C.-C.; Chung, L. W. Nat. Commun. 2020, 11, 1850 .

[46] Zheng, C. Chin. J. Chem. 2020, 38, 1579.

[47] Zheng, C.; Xia, Z.-L.; You, S.-L. Chem 2018, 4, 1952.

[48] Xie, J.; Otto, R.; Mikosch, J.; Zhang, J.; Wester, R.; Hase, W. L. Acc. Chem. Res. 2014, 47, 2960

[49] Xie, J.; Hase, W. L. Science 2016, 352, 32. 